\title{
Study on Dynamic Characteristics of Amphibious Aircraft's Water Landing
}

\author{
Xiao-Ming LIU 1,a , Yu-Tai HUANG ${ }^{2, b}$, Zhi-Cheng OU²,c , Xiao-Qing ZHANG, ${ }^{2, d}$ \\ ${ }^{1}$ Technology Center, Chengdu Aircraft Industrial (Group) Co., Ltd, Chengdu, 610092 \\ ${ }^{2}$ Department of Engineering Mechanics, School of Civil Engineering and Transportation ,South \\ China University of Technology, Guangzhou, 510640 \\ a694482374@qq.com, b511243865@qq.com, co.zc@qq.com, dtcqzhang@scut.edu.cn \\ ${ }^{*}$ Corresponding author
}

Keywords: Amphibious Aircraft, Fsi, Cel, Water Landing.

\begin{abstract}
The safety performance of water landing is an important research for the amphibious aircraft. The interactions between the airplane structure and water belong to complex fluid-structure interaction(FSI) problem. The three-dimensional model of water landing for a certain type of amphibious aircraft's head has been established. The structural dynamic response of the head's bottom has been analyzed by Coupled Euler-Lagrange(CEL). When the head landing into the water instantaneously, the pressure of the head's bottom appears peak value; with the secondary collision, the peak value appears again, and then the pressure decays gradually.
\end{abstract}

\section{Introduction}

The first amphibious aircraft was flight successfully by the French, Ferber, in 1910. With the development of technology and the increase of demand, amphibious aircraft came into being, which is a special seaplane that can take off and land on both land and water, so it highly adaptives to floods, forest fire suppression, emergency rescue, and many other needs. Currently, only a few countries such as the United States, China, Japan and Canada have the ability to develop amphibious aircraft.

The problem of water landing for the amphibious aircraft[1] is an important research content, and a typical problem of fluid-structure interaction[2], which is studied by experimental[3] and theoretical[4] early, with the development of computer, numerical simulation analysis[5] is also increasingly being used to solve the problem. In this paper, we base on ABAQUS FEM software and use CEL algorithm, consider the amphibious aircraft's head as rigid and elastomeric to make a comparison of their pressure firstly. Then consider the head as elastomers to analyze its stress and pressure.

\section{Coupled Eulerian-lagrange Algorithm}

Lagrange algorithm bases on the coordinates of substances, which divisions the described elements in the analyzed object. So the element mesh and the analyzed object of Lagrangian algorithm are uniform. While eulerian algorithm bases on the spatial coordinates, its elements independent of the analyzed objects. Throughout the process of simulation, the shape, size and spatial location of eulerian finite element mesh remain unchanged, but material in the elements is able to flow, that is to say, material can flows in a fixed field.

Article about Coupled Eulerian-Lagrange(CEL) is first proposed by Noh WF[6]. It is a new algorithm which is a combination of Lagrange algorithm and eulerian algorithm. In CEL, the mesh point can be moved together with material point, and can also be stationary in spatial, even can be fixed in one direction but moved together with material in other directions. For resolving the large deformation such as collision, fluid-structure interaction problem, CEL has great advantages.

\section{The Numerical Simulation of Water Landing}

\section{Water Landing Model}

Head's water landing model is shown in Figure 1. Head's skin and webs are made of four-node 
quadrilateral and three-node triangular shell elements, the hull reinforcement uses beam elements, and hull bottom is local cypher. Head's parts are made of aluminum whose material properties is described by bilinear elastic-plastic constitutive model.

Water model is an elliptical cylinder. Its size is $18 \mathrm{~m} \times 12 \mathrm{~m} \times 3 \mathrm{~m}$, elements type is the hexahedral unit of Euler. Consider splashing, Euler unit area slightly larger than the initial waters, so we set the depth of the initial waters $2.7 \mathrm{~m}$, and Euler unit is higher than the initial waters level $0.3 \mathrm{~m}$. In this paper, we use the linear $U s-U p$ Hugoniot equation of state to describe the characteristics of the water, where $s=\Gamma_{0}=0, c=1483 \mathrm{~m} / \mathrm{s}, \rho=1000 \mathrm{~kg} / \mathrm{m}^{3}$ and $\mu=0.001 \mathrm{~kg} /(\mathrm{m} \cdot \mathrm{s})$.

Considered $2 / 3 \mathrm{G}$ of upward pneumatic power, we set $1 / 3 \mathrm{G}$ for the head's gravity. Water apply gravity field. The boundary of water is free flow and non-reflective outflow. Head's horizontal velocity is $41 \mathrm{~m} / \mathrm{s}$ and vertical speed is $3 \mathrm{~m} / \mathrm{s}$, and diving angle is $7^{\circ}$. The entire calculation time is $0.1 \mathrm{~s}$.

\section{Model Comparison}

Head is considered rigid and elastomeric to analyze its dynamic response. When head lands into the water, the middle surface of bottom is contact with water firstly, so we select two models' middle surface for comparison of pressure, as shown in Figure 2, and the pressure time history curve is shown in Figure 3. When considering rigid model, the first wave of pressure appears in $0.002 \mathrm{~s}$, the pressure value is $105 \mathrm{kPa}$, and the second wave appears in $0.051 \mathrm{~s}$, the peak is $86 \mathrm{kPa}$. As a elastomeric, the first peak appears $0.002 \mathrm{~s}$, the pressure is $60 \mathrm{kPa}$, and second crest appears $0.053 \mathrm{~s}$, its peak is $65 \mathrm{kPa}$. Overall the pressure time history curve, two models have the same trend basically, the peak of pressure appears at the beginning, and a peak appears again after the second impact, then decay. But the force of rigid model is greater than elastomeric, and the peak of pressure appears earlier. To make the calculation more realistic, select elastomer model for the after study.

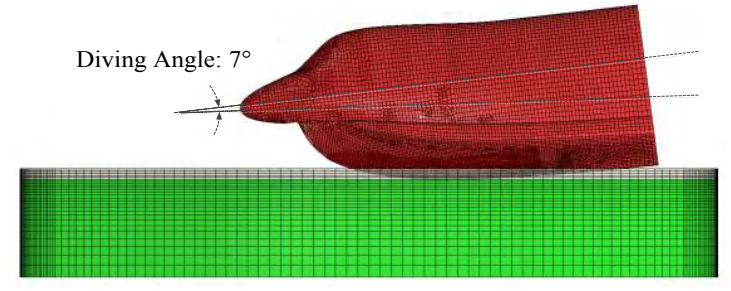

Fig. 1 Head's Water Landing Model

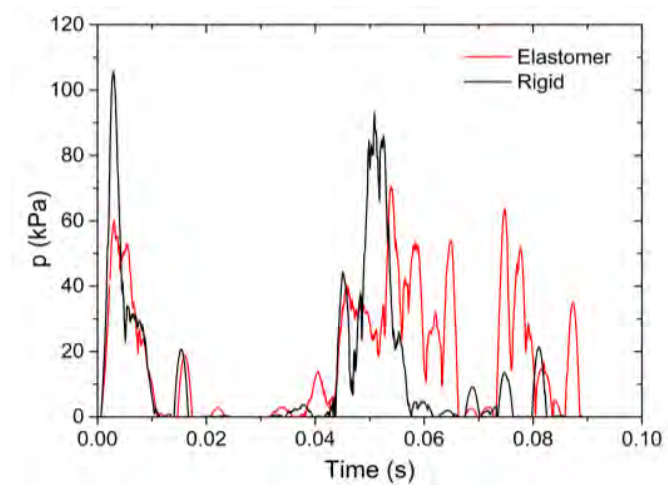

Fig. 3 Pressure of Elastomers and Rigid

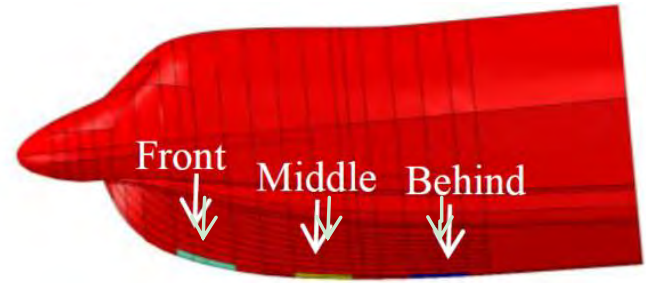

Fig. 2 Schematic of Bottom Surface

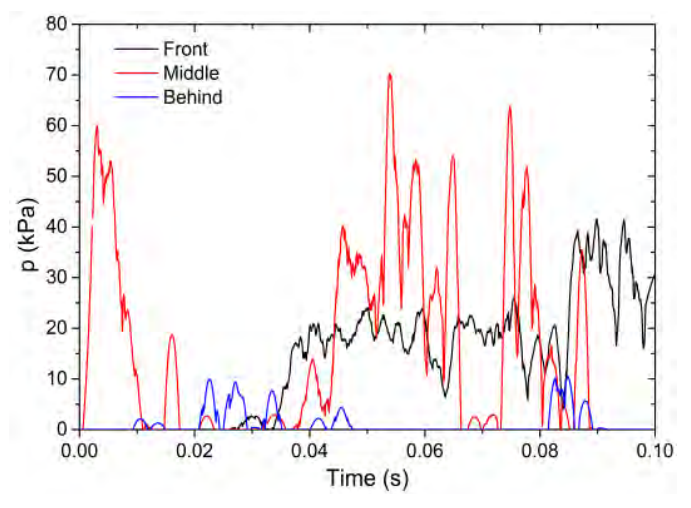

Fig. 4 Pressure-Time Curves of Surfaces

\section{Head's Stress and Waters}

The cloud picture of head bottom's stress and the deformation of water are shown in Table 1. At the beginning, head's bottom tangents to the water firstly, and contacts with water from 0s. The maximum stress occurs at the bottom at $0.014 \mathrm{~s}$, its value is $524 \mathrm{MPa}$. Waters appears splashing phenomenon. With the increase of depth in water, affected area of the hull increases, and scratches in the water gets deeper. In the whole process, the hull suffers the most significant effect and the maximum stress occurs at the beginning, then volatility decreases. 
Tab. 1 The Cloud Picture of Head's Stress and the Water

\begin{tabular}{|c|c|c|c|}
\hline Time(s) & Stress $(\mathrm{MPa})$ & Cloud Picture & Water \\
\hline 0.014 & 524 & $\ldots$ & 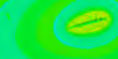 \\
\hline 0.050 & 490 & clings 8 & \\
\hline 0.075 & 501 & thes & \\
\hline
\end{tabular}

\section{Bottom's Pressure}

Select three surfaces of the bottom, front, middle and behind, to analyse the pressure, schematic is shown in Figure 2, and the pressure-time curves is shown in Figure 4. Middle surface in contact with water at the beginning and the pressure peak occurs at $0.003 \mathrm{~s}$, then the secondary impact occurs at $0.05 \mathrm{~s}$, the pressure peak occurs again, and the value slightly larger than the first impact, then slowly decays. With the increase sinking, the front and behind surfaces begin to contact with water, at the same time, the pressure of front surface increases and behind surface smaller impacts.

\section{Summary}

The finite element model of water landing for the head has established firstly. Then using the CEL analyzes the structural dynamic response of the head. Compared elastomer model, the force of rigid model greater and the pressure peak occurs earlier. The most obvious force occurs at the bottom, the maximum stress appears at the beginning, and then fluctuations decrease. The pressure peak appears at middle surface in the bottom at the initial time. With the secondary impact occurring, the peak occurs again, and its value slightly larger than the first impact, then slowly decays.

\section{References}

[1]Wang Yonghu. Review on research and development of water-entry impact problem,J. EXPLOSION AND SHOCK WAVES, 2008, Vol. 28, No. 3:276-282.

[2]Qian Ruojun. Advances in research on fluid structure interaction theory, J. SPATIAL STRUCTURES, 2008, Vol.14 No.1: 3-15.

[3]Bottomley G H. The impact of a model seaplane floats on water, R. Reports and Memoranda, No $583,1919$.

[4]Karman V. The impact on seaplane floats during landing, C. NACATN 321, 1929.

[5]Zhang Tao. Analysis Model and Numeral Simulation for Civil Plane Ditching, J. Journal of Nanjing University of Aeronautics \& Astronautics, 2010, Vol. 42 No. 3:392-394.

[6]Noh WF. CEL: A time-dependent two-space-dimensional coupled Eulerian-lagrangian code. In: B Alder, S Fernbach and M Rotenberg (eds.): Methods in Computational Physics, Volume 3,Fundamental Methods in Hydrodynamics. Academic Press, New York, NY, 1964, pp.117-179. 